\title{
An Ontology for Network Services
}

\author{
Pedro Alípio, José Neves, and Paulo Carvalho \\ Universidade do Minho, Departamento de Informática, \\ 4710-057 Braga, Portugal \\ \{pma, jneves, pmc\}@di.uminho.pt
}

\begin{abstract}
Most of the network service specifications use XML based models. However, as XML imposes a hierarchical structure, several types of relations may not be modeled. Therefore, richer specification languages are required in order to specify all network services vocabulary and how it relates with management tasks and with network configuration. This paper presents an ontology based model for network services, overcoming those semantic gaps and creating a better ground for reasoning over services fostering their self-configuration.
\end{abstract}

\section{Introduction}

Many network service management tasks such as service administration, service quality monitoring, service configuration, and resource optimization are often performed manually. This work can be time-consuming and very sensible to human errors. Moreover, it requires a growing number of highly skilled personnel, bringing huge costs to Internet Service Providers (ISPs).

Frequently, ISPs network services are expressed through Service Level Agreements (SLAs), where a technical part called Service Level Specification (SLS) is included. Several proposals of SLA and SLS specification have been presented, fostering a common ground for interoperability among domain and interdomain network service configuration agents. However, none of those specification is expressive enough to include the necessary knowledge to map service requirements into network configurations.

An ontology defines a common vocabulary for information interchange in a knowledge domain [1 and allows: (i) sharing common understanding of the structure of information among people or software agents; (ii) the reuse of domain knowledge; (iii) making domain assumptions explicit; (iv) separating domain knowledge from the operational knowledge; (iv) analysing domain knowledge 2 . This work suggests the use of an ontology for ISP network service specification instead of the traditional approaches.

This paper has the following structure: related work and the state-of-the-art in network service specification is presented in Section 2, the concepts and relations used to model service vocabulary and configuration mappings are explained in Section 3. finally, conclusions and future work are presented in Section 4 


\section{Related Work}

Network service specifications, apart from being a key aspect for QoS provisioning, provide a valuable input for network configuration. Therefore, defining a network service ontology, including SLA semantics and vocabulary is crucial for ensuring Quality of Service (QoS). Several working groups are committed to SLS definition and management [3, 4, 5, 6. Usually, XML is the preferred network services specification language. However, pure XML forces a hierarchical structure and it does not allow complex relations between objects.

Lately, ontologies are being used to bring semantics to the World-Wide Web (WWW). The WWW Consortium (W3C) developed the Resource Description Framework (RDF) 7], a language for encoding knowledge on Web pages to make it understandable to electronic agents searching for information. More recently, several ontology specification languages were developed with more expressive constructs aimed at facilitating agent interaction on the Web [8, 9,

Most of the ontology specification languages rely on XML and RDF only as underneath platform [8, 10, 9]. As a result, these ontologies may be validated, parsed or transformed with regular XML tools. Nevertheless, reasoning (queries, verification and taxonomical inference) is often performed by knowledge based systems that use other formalisms.

\section{Network Service Specification Ontology}

The main objective of the ontological representation of network services is to create a common vocabulary, including a service classification, and to map service attributes into network configurations. The model comprises three abstraction layers: (i) a higher level including the SLA and the service classification; (ii) a medium level including service level specification sections, as it is presented in [6]; (iii) a lower level, including the mappings to network configurations following the guidelines for Differentiated Services (Diffserv) [1].

\subsection{Service Classification}

Network traffic is classified in three groups: (i) Network Control for routing and network control function; (ii) Operations, Administration and Management (OAM) for network configuration and management functions; and (iii) the User/Subscriber traffic group for ISP functions which may be divided into nine different categories, namely: Telephony service; Signalling service; Multimedia Conferencing service; Real-time Interactive service; Multimedia Streaming service; Broadcast Video service; Low Latency Data service; High Throughput Data service; and Default service class.

Although User/Subscriber services are classified into nine groups, some are used by the same application category. In this model, four application categories are considered: (i) Application Control, including the Signalling service; (ii) Media-Oriented, including Telephony, Broadcast Video, Multimedia Conferencing and Real-time Interactive services; (iii) Data, including the Low Priority, 
Low Latency and High Throughput services; and (iv) Best Effort, including the Default Service. This ontology also includes a relationship between an SLA and a service specification, where each SLA may in fact include several different service classes, as some applications require signalling.

\subsection{Service Level Specification Sections}

The SLS ontology includes aggregation relations representing the following SLS sections: (i) the traffic classification section, defining the fields which identify an individual or aggregate flow; (ii) the traffic conditioning section, containing rules to identify in or out-of-profile traffic; (iii) the scope of the service, defining the boundaries of the region over which the service will be enforced; (iv) the expected QoS performance parameters; (v) the service scheduling section, defining the time period when the service is available; and (vi) the service reliability section, defining parameters related to the consistency and reliability of the service to be provided.

\subsection{Network Configuration}

This level of the ontology model should be observed as node centric instead of service centric as configurations are in fact performed on the nodes. In the ISP network there are two main types of nodes: edge nodes (Ingress and Egress), and core nodes. Edge nodes are far more complex as they include policers and classifiers, while core nodes just assure that the Per-Hop Behaviour (PHB) specified by the ingress is kept for each packet. As a result, the following configurations are possible: (i) configuration of the queueing disciplines; (ii) configuration of queue congestion control, and (iii) mapping classes into queues.

Associated to a node link, there may be both priority queueing and rate queueing disciplines. A priority queueing system is a combination of a set of queues and a scheduler that empties them in priority sequence. When asked for a packet, the scheduler inspects the highest priority queue, and if there is data present returns a packet from that queue. Similarly, a rate-based queueing system is a combination of a set of queues and a scheduler that empties each at a specified rate. Each queue is associated to a DiffServ Code Point (DSCP) and some queues may have congestion control trough Active Queue Management (AQM) consisting of a variety of procedures that use packet dropping or marking to manage the depth of a queue.

\section{Conclusion and Future Work}

This work intends to go much further than a service specification. Service specifications usually include several sections, which describe the service requirements with different technical perspectives: traffic classification, traffic conditioning, scope, expected QoS, scheduling and service reliability. However, those specifications never include network configuration and the related information. In those approaches, mapping services into network configurations cannot be done 
conceptually, because XML is often used and it imposes a hierarchical structure, which is not adequate to specify complex relations between services and network devices. By modeling network services in terms of an ontology, those limitations are overcome. Moreover, several classes and relations may not be explicitly defined, as they may be deducted through inference rules.

Work is currently in progress to create a service specification beyond its technical aspects, involving the administrative and management perspectives.

Acknowledgements. A PhD grant provided by Fundação para a Ciência e Tecnologia (SFRH/ BD/ 17579/2004) is gratefully acknowledged.

\section{References}

1. Gruber, T.R.: Toward principles for the design of ontologies used for knowledge sharing. Int. J. Hum.-Comput. Stud. 43 (1995) 907-928

2. Noy, N.F., McGuinness, D.L.: Ontology Development 101: A Guide to Creating Your First Ontology. Stanford Knowledge Systems Laboratory Technical Report KSL-01-05 and Stanford Medical Informatics Technical Report SMI-2001-0880 (2001)

3. Morand, P., Boucadair, M., P. Levis, R.E., Asgari, H., Griffin, D., Griem, J., Spencer, J., Trimintzios, P., Howarth, M., Wang, N., Flegkas, P., Ho, K., Georgoulas, S., Pavlou, G., Georgatsos, P., Damilatis, T.: Mescal D1.3 - Final Specification of Protocols and Algorithms for Inter-domain SLS Management and Traffic Engineering for QoS-based IP Service Delivery and their Test Requirements . Mescal Project IST-2001-37961 (2005)

4. Diaconescu, A., Antonio, S., Esposito, M., Romano, S., Potts, M.: Cadenus D2.3 Resource Management in SLA Networks. Cadenus Project IST-1999-11017 (2003)

5. Alipio, P., Lima, S., Carvalho, P.: XML Service Level Specification and Validation. In: 10th IEEE Symposium on Computers and Communications (ISCC'05). (2005) 975-980

6. Goderis, D., T'joens, Y., Jacquenet, C., Memenious, G., Pavlou, G., Egan, R., Griffin, D., Georgatsos, P., Georgiadis, L., Heuven, P.V.: Service Level Specification Semantics, Parameters, and Negotiation Requirements. Internet-Draft, drafttequila-sls-03.txt (work in progress) (2003)

7. Brickley, D., Guha, R.: Resource Description Framework (RDF) Schema Specification. http://www.w3.org/TR/rdf-schema, W3C (1999)

8. Hendler, J., McGuinness, D.: Darpa agent markup language. IEEE Intelligent Systems 15 (2000)

9. Bechhofer, S., van Harmelen, F., Hendler, J., Horrocks, I., McGuinness, D.L., PatelSchneider, P.F., , Stein, L.A.: OWL Web Ontology Language Reference. W3C (2004)

10. Connolly, D., van Harmelen, F., Horrocks, I., McGuinness, D.L., Patel-Schneider, P.F., Stein, L.A.: DAML+OIL Reference Description. W3C (2001)

11. Babiarz, J., Chan, K., Baker, F.: Configuration Guidelines for DiffServ Service Classes. Internet Draft (work in progress) (2005) 